Instrumental Achievements

- X-Ray Analysis -

\title{
Crystal Structure of Arsenazo I
}

\author{
Michio ZenKI*, Takashi Shibahara*, Mikio YamaSAKI* and Yoshihiko KUSHI** \\ *Department of Chemistry, Faculty of Science, Okayama University of Science, Okayama 700, Japan \\ ** College of General Education, Osaka University, Toyonaka, Osaka 560, Japan
}

Arsenazo I (Neothorin), 3-[(2-arsonophenyl)azo $]-4,5-$ dihydroxy-2,7-naphthalenedisulfonic acid has been known as a sensitive reagent for $\mathrm{Th}, \mathrm{Zr}$ and alkaline earth metals. ${ }^{1,2}$ Our main interest lies in the reactions of mono- or bisarylazo chromotropic acid derivatives with various metal ions. As a part of serial studies, an $\mathrm{X}$-ray analysis of Arsenazo I has been undertaken.

A red-orange needle crystal $(0.34 \times 0.19 \times 0.12 \mathrm{~mm})$, grown from $2 \mathrm{M} \mathrm{HCl}$ solution, was used for the $\mathrm{X}$-ray study. As shown in Fig. 2, the molecule is very nearly planar. The distances, OH1-OH2, OH1-N1, OH1OA2 and N1-OA2 were 2.55(2), 2.57(2), 2.85(2) and 3.05(1) $\AA$, respectively; it can be assumed that three intramolecular hydrogen bondings exist as indicated in Fig. 1. The abnormally short distances SB2-OSB are due to the large thermal vibrations. All of the waters of crystalization were disordered (Table 2). The structure of metal chelates with this reagent will be reported elsewhere.<smiles>O=C(O)c1ccccc1N=Nc1c(S(=O)(=O)O)cc2cc(S(=O)(=O)O)cc(O)c2c1O</smiles>

Fig. I Chemical structure.
Table 1 Crystal and experimental data

Formula: $\mathrm{C}_{16} \mathrm{H}_{13} \mathrm{~N}_{2} \mathrm{O}_{11} \mathrm{~S}_{2} \mathrm{As} \cdot 3 \mathrm{H}_{2} \mathrm{O}$

Formula weight $=602.34$

Crystal system: triclinic

Space group: $P \overline{1} \quad Z=2$

$a=14.880(6) \AA \quad \alpha=89.01(3)^{\circ}$

$b=12.245(4) \AA \quad \beta=99.63(3)^{\circ}$

$c=7.338(3) \AA \quad \gamma=101.54(3)^{\circ}$

$V=1291.4(8) \AA^{3}$

$D_{\mathrm{x}}=1.549 \mathrm{~g} / \mathrm{cm}^{3}$

$R=0.071$

No. of reflections used $=2570$

Measurement: Rigaku AFC-6A

Program system: UNICS II

Structure determination: heavy atom method

Refinement: block-diagonal least-squares

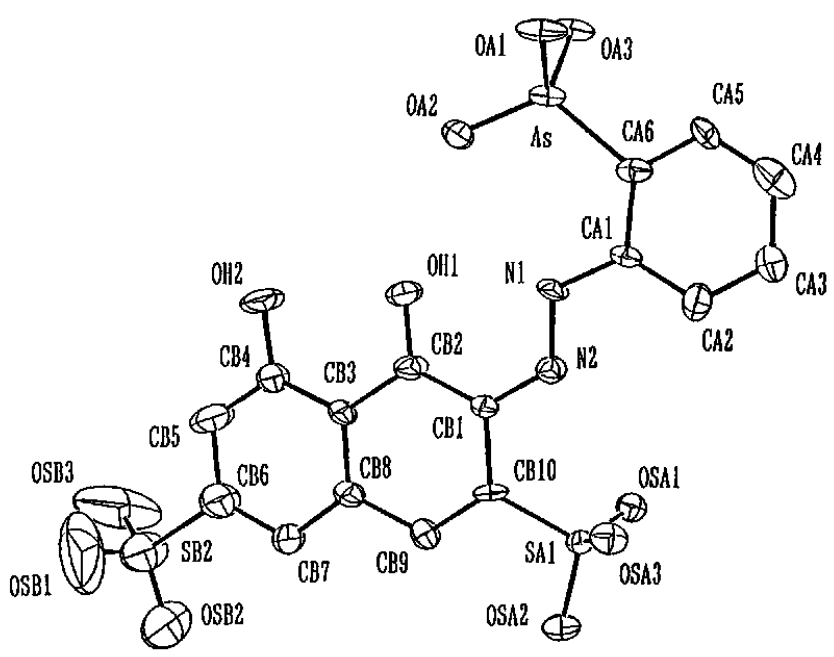

Fig. 2 A perspective view with atom numbering. 
Table 2 Final coordinates of non-H atoms

\begin{tabular}{|c|c|c|c|c|}
\hline & $x$ & $y$ & $z$ & $B_{e q} / \AA^{2}$ \\
\hline AS & $0.1108(1)$ & $1.3665(1)$ & $0.2894(2)$ & $2.67(3)$ \\
\hline SA 1 & $-0.0701(2)$ & $0.7487(2)$ & $0.2259(4)$ & $2.3(1)$ \\
\hline $\mathrm{OA} 1$ & $0.1192(7)$ & $1.4608(6)$ & $0.115(1)$ & $4.2(3)$ \\
\hline $\mathrm{OA} 2$ & $0.2010(5)$ & $1.3026(6)$ & $0.318(1)$ & $3.2(2)$ \\
\hline OA3 & $0.1189(6)$ & $1.4607(5)$ & $0.463(1)$ & $3.6(3)$ \\
\hline OSA 1 & $-0.1076(5)$ & $0.7812(6)$ & $0.379(1)$ & $2.9(2)$ \\
\hline $\mathrm{OSA} 2$ & $-0.0764(5)$ & $0.6247(5)$ & $0.222(1)$ & $3.1(2)$ \\
\hline OSA 3 & $-0.1101(5)$ & $0.7845(6)$ & $0.045(1)$ & $3.1(2)$ \\
\hline $\mathrm{OH} \mathbf{l}$ & $0.2130(6)$ & $1.0728(6)$ & $0.324(2)$ & $5.4(3)$ \\
\hline $\mathrm{OH} 2$ & $0.3797(6)$ & $1.0413(7)$ & $0.388(2)$ & $8.7(5)$ \\
\hline N1 & $0.0434(6)$ & $1.0984(6)$ & $0.269(1)$ & $2.6(3)$ \\
\hline N2 & $0.0190(6)$ & $0.9869(7)$ & $0.258(1)$ & $2.5(3)$ \\
\hline CA1 & $-0.0290(8)$ & $1.1550(8)$ & $0.244(2)$ & $2.5(3)$ \\
\hline $\mathrm{CA} 2$ & $-0.1226(8)$ & $1.102(1)$ & $0.209(2)$ & $3.7(4)$ \\
\hline $\mathrm{CA} 3$ & $-0.1928(9)$ & $1.164(1)$ & $0.184(2)$ & $4.4(4)$ \\
\hline $\mathrm{CA} 4$ & $-0.169(1)$ & $1.281(1)$ & $0.188(2)$ & $5.1(5)$ \\
\hline CA5 & $-0.0792(8)$ & $1.3335(9)$ & $0.221(2)$ & $3.4(4)$ \\
\hline CA6 & $-0.0112(8)$ & $1.2733(8)$ & $0.247(2)$ & $2.8(3)$ \\
\hline CB1 & $0.0826(7)$ & $0.9249(8)$ & $0.277(2)$ & $2.3(3)$ \\
\hline CB2 & $0.1810(8)$ & $0.9691(8)$ & $0.312(2)$ & $3.2(4)$ \\
\hline CB3 & $0.2466(8)$ & $0.8929(8)$ & $0.336(2)$ & $3.1(4)$ \\
\hline CB4 & $0.3413(8)$ & $0.9315(9)$ & $0.376(3)$ & $5.3(5)$ \\
\hline CB5 & $0.403(1)$ & $0.861(1)$ & $0.407(3)$ & $7.2(7)$ \\
\hline CB6 & $0.365(1)$ & $0.743(1)$ & $0.386(3)$ & $6.5(6)$ \\
\hline CB7 & $0.2696(8)$ & $0.700(1)$ & $0.349(2)$ & $4.7(5)$ \\
\hline CB8 & $0.2113(8)$ & $0.7774(8)$ & $0.322(2)$ & $3.1(4)$ \\
\hline CB9 & $0.1091(8)$ & $0.7338(9)$ & $0.282(2)$ & $3.2(4)$ \\
\hline CB10 & $0.0477(8)$ & $0.8045(7)$ & $0.263(2)$ & $2.4(3)$ \\
\hline SB2 & $0.4424(3)$ & $0.6511(4)$ & $0.424(1)$ & $11.1(3)$ \\
\hline OSB 1 & $0.489(2)$ & $0.669(1)$ & $0.272(5)$ & $27 .(2)$ \\
\hline OSB2 & $0.3916(8)$ & $0.5463(9)$ & $0.412(3)$ & $13.1(8)$ \\
\hline OSB3 & $0.509(1)$ & $0.692(1)$ & $0.565(5)$ & $26 .(2)$ \\
\hline OW 1A & $0.690(3)$ & $0.797(3)$ & $0.328(6)$ & $8 .(1)^{a}$ \\
\hline OW 1B & $0.690(1)$ & $0.700(2)$ & $0.385(3)$ & $9.7(5)^{a}$ \\
\hline OW $2 A$ & $0.700(2)$ & $0.769(3)$ & $-0.042(5)$ & $9.3(9)^{\mathrm{a}}$ \\
\hline OW 2B & $0.717(2)$ & $0.850(2)$ & $0.112(4)$ & $11.8(8)^{a}$ \\
\hline OW $3 A$ & $0.627(3)$ & $0.585(4)$ & $-0.147(8)$ & $16 .(2)^{a}$ \\
\hline OW 3B & $0.675(2)$ & $0.668(2)$ & $-0.152(5)$ & $14.0(9)^{a}$ \\
\hline
\end{tabular}

$B_{e q}=(4 / 3) \Sigma_{i} \Sigma_{j} \beta_{i j} a_{i} a_{j}$.

a. Isotropic temperature factors were applied. The following occupancy factors were used for the disordered atoms: OW1A, 0.3; OW 1B, 0.7; OW2A, 0.4; OW2B, 0.6; OW 3A, 0.4; OW3B, 0.6.
Table 3 Bond distances $(\AA)$ and angles $\left(^{\circ}\right)$

\begin{tabular}{|c|c|c|c|c|c|}
\hline$A S-O A 1$ & \multicolumn{2}{|c|}{$1.713(8)$} & $\mathrm{CA} 4-\mathrm{CA} 5$ & \multicolumn{2}{|c|}{$1.345(18)$} \\
\hline$A S-O \wedge 2$ & \multicolumn{2}{|c|}{$1.667(8)$} & CA5-CA6 & \multicolumn{2}{|c|}{ J. $353(18)$} \\
\hline$A S-O A 3$ & \multicolumn{2}{|c|}{$1.705(8)$} & $\mathrm{CB} 1-\mathrm{CB} 2$ & \multicolumn{2}{|c|}{$1.437(15)$} \\
\hline$A S-C \wedge 6$ & \multicolumn{2}{|c|}{$1.921(11)$} & $\mathrm{CB} 1-\mathrm{CB} 10$ & \multicolumn{2}{|c|}{$1.461(13)$} \\
\hline $\mathrm{SAl} 1-\mathrm{OSA} \mathrm{I}$ & \multicolumn{2}{|c|}{$1.434(9)$} & $\mathrm{CB} 2-\mathrm{CB} 3$ & \multicolumn{2}{|c|}{$1.468(17)$} \\
\hline $\mathrm{S} \wedge 1--0 \mathrm{OS} \wedge 2$ & \multicolumn{2}{|c|}{$1.502(7)$} & CB3-CB1 & \multicolumn{2}{|c|}{$1.377(16)$} \\
\hline $\mathrm{S} A 1-\mathrm{OSA} 3$ & \multicolumn{2}{|c|}{$1.461(8)$} & CB $3-\mathrm{CB8}$ & \multicolumn{2}{|c|}{$1.406(11)$} \\
\hline$S A 1-C B 10$ & \multicolumn{2}{|c|}{$1.727(11)$} & $\mathrm{CB} 4-\cdots \mathrm{CB5}$ & \multicolumn{2}{|c|}{$1.375(20)$} \\
\hline $\mathrm{OH} 1-\mathrm{CB} 2$ & \multicolumn{2}{|c|}{$1.263(12)$} & CB5-CB6 & \multicolumn{2}{|c|}{$1.442(18)$} \\
\hline $\mathrm{OH} 2-\mathrm{C} B 4$ & \multicolumn{2}{|c|}{$1.350(13)$} & $\mathrm{CB} 6-\mathrm{CB} 7$ & \multicolumn{2}{|c|}{ 1. $392(18)$} \\
\hline $\mathrm{N} 1-\mathrm{N} 2$ & \multicolumn{2}{|c|}{$1.341(11)$} & $\mathrm{CB} 6-\mathrm{SB} 2$ & \multicolumn{2}{|c|}{$1.750(16)$} \\
\hline $\mathrm{N} 1-\mathrm{C} \Lambda 1$ & \multicolumn{2}{|c|}{$1.380(15)$} & $\mathrm{CB} 7-\mathrm{CB} 8$ & \multicolumn{2}{|c|}{$1.396(18)$} \\
\hline $\mathrm{N} 2-\mathrm{CB} 1$ & \multicolumn{2}{|c|}{$1.316(15)$} & $\mathrm{CB} 8-\mathrm{CB} 9$ & \multicolumn{2}{|c|}{$1.488(16)$} \\
\hline $\mathrm{CA} 1-\mathrm{CA} 2$ & \multicolumn{2}{|c|}{$1.398(15)$} & $\mathrm{CB} 9-\mathrm{CB} 10$ & 1.3 & $9(17)$ \\
\hline $\mathrm{CA} 1-\mathrm{C} \wedge 6$ & 1.4 & $9(13)$ & SB2-OSB1 & 1.3 & $4(37)$ \\
\hline $\mathrm{CA2}-\mathrm{CA} 3$ & 1.3 & $9(19)$ & $\mathrm{SB} 2-\mathrm{OSB} 2$ & 1.3 & $8(11)$ \\
\hline $\mathrm{CA} 3-\mathrm{CA} 4$ & 1.4 & $2(18)$ & $\mathrm{SB} 2-\mathrm{OSB} 3$ & & $7(25)$ \\
\hline $\mathrm{OAl}-\mathrm{AS}-$ & $\mathrm{OA} 2$ & $110.2(1)$ & $\mathrm{O} 111-\mathrm{CB2}$ & $\mathrm{CB} 1$ & $121.5(10)$ \\
\hline$O A 1-A S-$ & $0 \wedge 3$ & $96.7(4)$ & $\mathrm{O} 11 \mathrm{1}-\mathrm{CB} 2$ & CB3 & $118.7(10)$ \\
\hline$O A 1-A S-$ & CA6 & $108.8(4)$ & $\mathrm{CB} 1-\mathrm{CB} 2$ & CB3 & $119.8(9)$ \\
\hline $\mathrm{OA} 2-\mathrm{AS}-$ & OA3 & $112.1(4)$ & $\mathrm{CB} 2-\mathrm{CB} 3$ & BA & $121.8(9)$ \\
\hline $\mathrm{OA} 2-\mathrm{AS}$ & CAG & $117.0(4)$ & $\mathrm{CB} 2-\mathrm{CB} 3$ & CB8 & $118.8(10)$ \\
\hline$O A 3-A S$ & CA6 & $110.1(5)$ & $\mathrm{CB} 4=\mathrm{CB} 3$ & CB8 & $119.3(11)$ \\
\hline $\mathrm{N} 2-\mathrm{N}_{1}-$ & $\mathrm{CAl}$ & $115.8(8)$ & $\mathrm{OH} 2-\mathrm{CB} 4$ & B3 3 & $122.2(11)$ \\
\hline $\mathrm{N} 1-\mathrm{N} 2-$ & $\mathrm{CB} 1$ & $120.8(8)$ & $\mathrm{OH} 2-\mathrm{CB} 4$ & CB5 & $115.6(11)$ \\
\hline $\mathrm{N} 1-\mathrm{CAl}$ & $\mathrm{CA} 2$ & $123.2(9)$ & $\mathrm{CB} 3-\mathrm{CB} 4$ & CB5 & $122.2(11)$ \\
\hline $\mathrm{N} 1-\mathrm{C} \wedge 1$ & CA 6 & $120.6(9)$ & $\mathrm{CB} A-\mathrm{CB} 5$ & $2 B 6$ & $117.2(12)$ \\
\hline $\mathrm{CA} 2-\mathrm{CA} 1$ & $\mathrm{c} \wedge 6$ & $116.1(11)$ & $\mathrm{CB} 5-\mathrm{CB} 6$ & CB7 & $122.3(14)$ \\
\hline $\mathrm{CA} 1-\mathrm{CA} 2$ & $\mathrm{CA} 3$ & $120.4(11)$ & $\mathrm{CB} 5-\mathrm{CB} 6$ & $\mathrm{~B} 2$ & $118.0(11)$ \\
\hline $\mathrm{CA} 2-\mathrm{CA} 3$ & CA 4 & $119.5(12)$ & $\mathrm{CB} 7-\mathrm{CB} 6$ & $6 B 2$ & $119.6(10)$ \\
\hline $\mathrm{CA} 3-\mathrm{C} A 4$ & CA 5 & $120.9(14)$ & $\mathrm{CB} 6-\mathrm{CB} 7$ & 138 & $117.1(11)$ \\
\hline CA4 $-\mathrm{CA} 5$ & $\mathrm{C} \Lambda 6$ & $119.6(11)$ & CB3-CB 8 & B 7 & $121.8(10)$ \\
\hline $\mathrm{AS}-\mathrm{C} \Lambda 6$ & $\mathrm{CAI}$ & $124.4(9)$ & $\mathrm{CH} 3-\mathrm{CB} 8$ & B9 & $120.3(10)$ \\
\hline $\mathrm{AS}-\mathrm{CA6}$ & CA5 & $112.1(7)$ & $\mathrm{CB} 7-\mathrm{CB} 8$ & ¿B9 & $117.9(9)$ \\
\hline $\mathrm{CAl}=\mathrm{CAC}$ & CA5 & $123.4(10)$ & CB8-CB9 & B 10 & $121.0(9)$ \\
\hline $\mathrm{N} 2-\mathrm{CB} 1$ & $\mathrm{CB} 2$ & $123.9(9)$ & $\mathrm{SA} 1-\mathrm{CB} 1$ & B 1 & $121.3(9)$ \\
\hline $\mathrm{N} 2-\mathrm{CB} 1$ & CB 10 & $115.9(9)$ & $\mathrm{SA1}-\mathrm{CB} 1$ & B9 & $118.9(7)$ \\
\hline CB2-CB1 & CB 10 & $120.2(10)$ & $\mathrm{CB} 1-\mathrm{CB} 1$ & CB9 & $119.8(10)$ \\
\hline
\end{tabular}

\section{References}

1. B. Budesinsky, "Chelates in Analytical Chemistry", ed. H. A. Flaschka and A. J. Barnard, Jr., Vol. 2, p. 1, Marcel Dekker, New York, 1969.

2. K. L. Cheng, K. Ueno and T. Imamura, "Handbook of Organic Analytical Reagents", p.159, CRC Press, Boca Raton, 1982. 